\title{
How do I track Cardiac Rehabilitation in my patient with ischemic heart disease using Strava
}

\author{
Oana Moldovan, Balasz Deak, Alin Bian, Diana Gurzau, Florina Frangu, Alexandru Martis, \\ Bogdan Caloian, Horatiu Comsa, Gabriel Cismaru, Dana Pop
}

Corresponding author: Gabriel Cismaru,E-mail: gabi_cismaru@yahoo.com ,

\begin{abstract}
Cardiac rehabilitation is a program which reduces morbidity and mortality in patients with ischemic heart disease. However only some European centers provide these programs for patients diagnosed with cardiac ischemia. Therefore home-based programs were developed for cardiac rehabilitation. Strava is a social network developed to track activities of running and cycling both in athletes and individuals searching for recreation and sport activities. In our work we present an approach based on Strava tracking to improve the quality of cardiac rehabilitation. Real-time monitoring of heart rate, steps per minute and speed during recreational or competitive activities can be used to increase physical capacity in individuals with ischemic heart disease.
\end{abstract}

Key words: cardiac rehabilitation, Strava, ischemic heart disease, home-based,

\section{Introduction}

Cardiac rehabilitation is the sum of actions needed to provide an optimal level of physical activity after an acute episode of cardiac disease. Beside risk factors, physical exercise is the cornerstone of rehabilitation (1).

\subsection{Cardiac rehabilitation in ischemic heart disease.}

Patients with acute coronary syndrome or post-PCI have a class I level A indication for cardiac rehabilitation in current guidelines, and patients with chronic angina have a class I level B indication (2). Physical training has been shown to reduce risk factors in patients with ischemic heart disease (3). Patients that had an acute myocardial infarction or an acute coronary syndrome, or underwent a coronary bypass procedure are eligible for cardiac rehabilitation programs. If the patient already developed NYHA IV heart failure or the coronarography shows significant remaining lesions or significant ventricular arrhythmias, cardiac rehabilitation could be postponed or even excluded as a way of treatment (4).

\subsection{Phases of Cardiac rehabilitation in ischemic} heart disease.

Cardiac rehabilitation programs consist of 3 phases. The first phase starts early after stent implantation, or medical treatment, in hospital, after assesing the basic functional mobility of the patient and associated risk factors. Upon discharge the patient can continue phase 2 consisting in monitored excercise activities.
The last phase is phase 3 with increased intensity of excercise guided by self- monitoring of the heart rate, cadence, and pace and self-perceived exertion $(5,6)$.

\subsection{Heart rate during physical training.}

Patients after an acute coronary event have an accelerated heart rate increase to standardized workload, similar to individuals with prolonged bed rest. This is called a deconditioning response to disease. The cardiac response to excercise is related to a decrease in the vagal tone which is further translated to an increase in the heart rate (7). During dynamic tpes of exercise heart rate increase is liniar to workload and within minutes it reaches a steady state for a given intensity of physical activity. Heart rate during excercise depends on the age of the patient, body position, type of physical activity, environment, and can be measured using different devices or apps (fig 1,2).

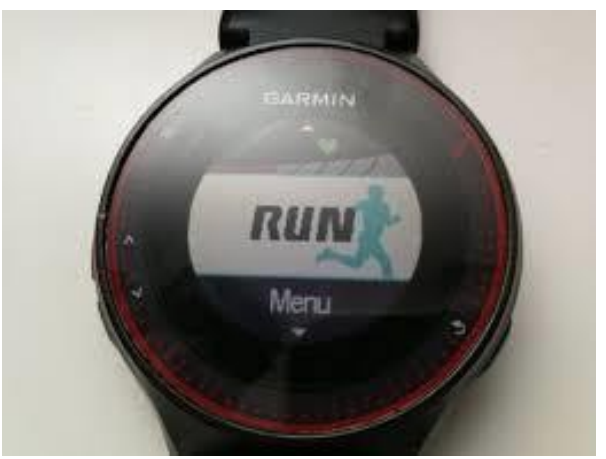

Fig. 1. Garmin Forrunner device used for excercise tracking. It can display distance, heart rate and pace. It uses an optical heart rate sensor with green light on the back of the device to measure heart rate. 


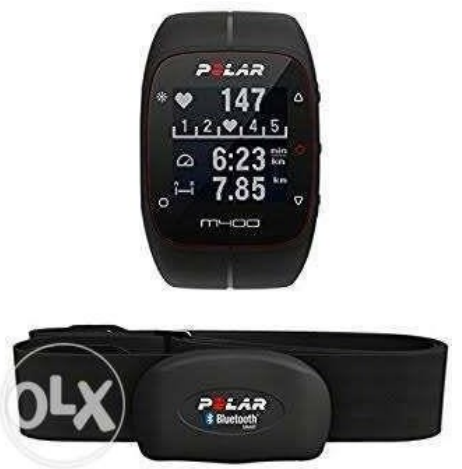

Fig. 2. Polar device used for excercise tracking. It has a pectoral belt for heart rate measurement that transmits the signal to the watch using bluetooth technology.

\section{Devices for home-based rehabilitation.}

Phase 3 rehabilitation is an alternative to cardiac rehabilitation programs that take place in hospitals or as out-patient rehabilitation. Home based rehabilitation may be improved using devices that measure not only biological variables such as heart rate, blood pressure, respiratory rate and caloric count but also cadence, speed and distance using motion sensors and accelerometers with long-term information storage programs. All those devices increase the adherence of patients to cardiac rehabilitation programs. A high number of devices for home-use are launched and improved every month to increase the ease of access to remote monitoring. It is possible in our days to assess home- based cardiac rehabilitation with real-time remote monitoring using various apps and devices making the follow-up of patients more efficient (8).

Polar and Garmin devices are tools for exercise tracking that can be used for home-based cardiac rehabilitation programs. They can be used by cardiologists to evaluate their patient's activity and keep track of the progress during the excercise program. Both Garmin and Polar devices include a wide range of watches of various size and style with or without an attached chest band for respiratory and heart rate monitoring during exercise. Devices can monitor physical activities like walking, running, swimming, bike riding and much more (9).

\subsection{Strava platform}

Strava platform has an important role in the quality of the rehabilitation program and patient's adherence. Doctors can obtain information both during excercise and postexcercise: activity duration, speed, average pace, elevation gain, calories burnt as well as heart rate and respiratory rate at specific splits during excercise, related to distance, pace and elevation. Strava automatically processes diagrams and figures based on measured parameters. This makes cardiac rehabilitation significantly easier for the patient and user friendly for the doctor that supervises the program from the hospital or his own computer (10).

Another important asset that this platform provides is the social network interface that can help increase the adherence to the program by offering the possibility to join various running clubs even cardiovascular rehabilitation clubs which can offer further support and motivation for the patient.

\subsection{Individual coaching by telephone or internet}

The telehealth or e-health technologies due to their lower grade of flexibility and professional input on excercise programs may not be as effective as the phase II-hospital based rehabilitation program. Nevertheless, the results of remote excercise monitoring can be improved by the real time advices of a cardiologist during the rehabilitation program, by phone, email or phone messages using different applications. Interaction with a cardiologist is expected to increase both the patient's adherence to the program and quality of training (11).

\section{Stepwise approach to Strava tracking}

Step 1: Before beginning stage 2 of cardiac rehabilitation a stress test should be performed to assess the maximum heart rate during physical activity. If the test is not performed the theoretical maximum heart rate will be calculated using the formula 220 -age.

Step 2: The patient's risk factors will be assesed: lowrisk, high-risk or very high-risk. High-risk patients are considered those with ejection fraction of $<40 \%$, patients with failed reperfusion therapy, patients with severe three-vesel disease and those $>70$ years old. Very high-risk patients are considered those that already have clinical signs of heart failure NYHA III of IV. 
Step 3: The patient should aquire a Strava compatible Step 6: Each time the patient performs physical activity, tracking device: Garmin, Polar (fig 1,2), Suunto, information will be uploaded to Strava and can be seen Tomtom, Fitbit and synchronize the device with the by the cardiologist for further advices.

Strava personal account.

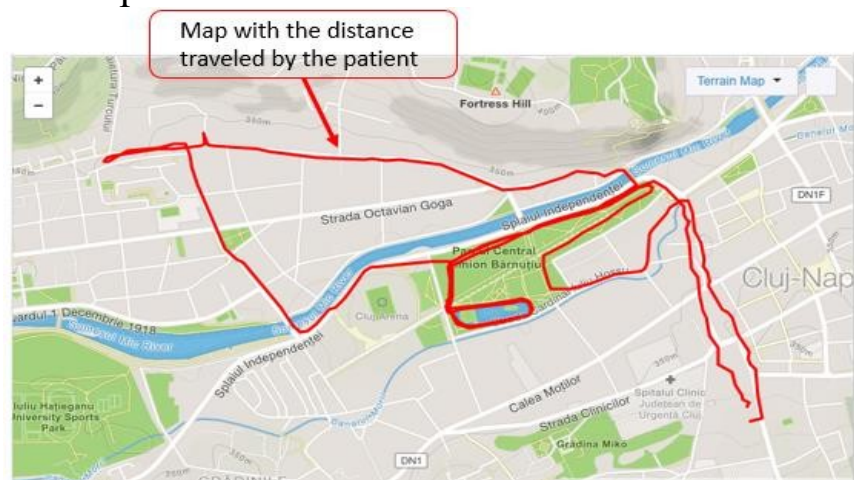

Fig. 3. Map with the distance traveled by the patient. The GPS system of the Garmin or Polar watch can trace the patient while exercising. (Source: personal collection)

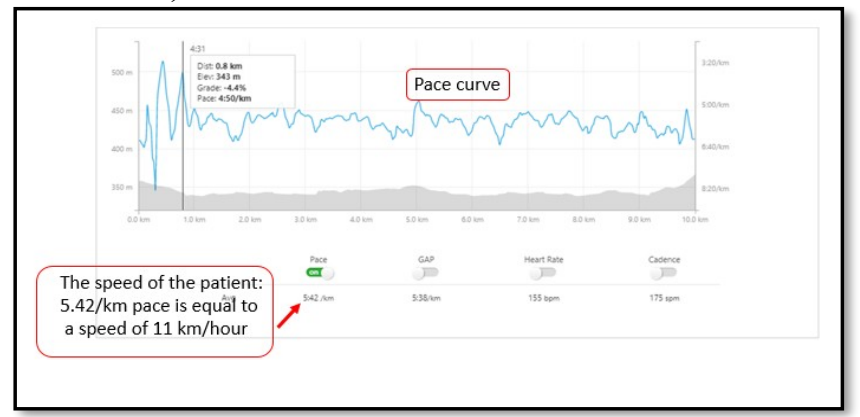

Fig. 4. After terminating the daily training program, the patient can upload his activity on Strava. This image shows the pace curve as minutes/ $\mathrm{km}$ during the total duration of 50 minutes training.

(Source: personal collection)

Step 4: The cardiologist will establish thre excercise intensity according to the risk, using the Karvonen formula:

Low risk $=0.6 \mathrm{x}(\max$ heart rate-resting heart rate)+resting heart rate.

Hish risk=-0.5 x (max heart rate-resting heart rate)+resting heart rate.

Very hisgh risk $=0.4 \mathrm{x}$ ( $\max$ heart rate-resting heart rate)+resting heart rate.

Step 5: The patient will start training with a frequency of 4 times/week for 30 minutes then will increase gradually to 7 times/week and up to 1 hour.
Step 7: The cardiologist can check the activities uploaded by his patients from his office or from his own home. He will search for: distance traveled by the athlete, speed, cadence and heart rate, different graphs and maps (Fig 3-6).

\section{Step 8:}

After verifying the data provided by Strava platform, the doctor can give advices on how to increase or decrease distance, speed and frequency of activities/day.

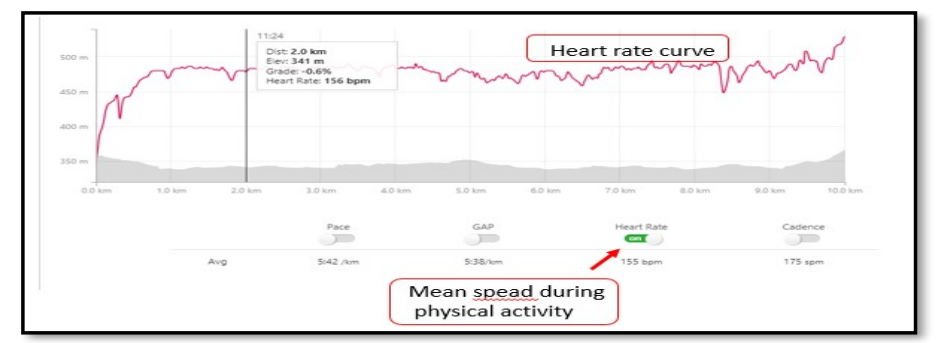

Fig. 5. Heart rate curve. For a rehabilitation cardiologist this curve is very important as it shows the intensity of physical activity performed by the patient. The patient did more intense activity than calculated by the Karvonen formula: for a 40- year-old patient is: (18080) $\mathrm{x} 0,6+80=140 \mathrm{bpm}$.

(Source: personal collection)

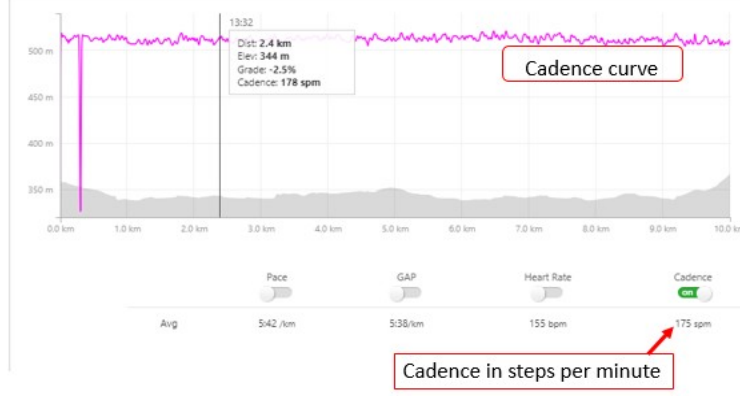

Fig. 6. Cadence curve. The figure shows the number of steps the patient takes per minute. In this case $175 \mathrm{spm}$ is a good cadence. (Source: personal collection) 


\section{Conclusion:}

Exercise training remains the cornerstone of rehabilitation in cardiovascular patients as it decreases morbidity and mortality in patients with ischemic heart disease, it improves symptoms and cardiac risk factors, as well as increases the quality of life by increasing physical functioning and exercise capacity. Strava, as a social network, using Polar and Garmin tracking devices, helps the patient with ischemic heart disease by improving the quality of the rehabilitation and increases the adherence to home based cardiac rehabilitation program

\section{Conflict of interest}

There is no conflict of interest for any of the authors regarding this paper.

\section{References:}

1. Fletcher GF, Balady G, Blair SN, et al. Statement on exercise: benefits and recommendations for physical activity programs for all Americans: a statement for health professionals by the Committee on Exercise and Cardiac Rehabilitation of the Council on Clinical Cardiology, American Heart Association. Circulation. 1996;94:857-862.

2. Fletcher GF, Balady G, Froelicher VF, et al. Exercise standards: a statement for healthcare professionals from the American Heart Association Writing Group. Circulation. 1995;91:580-615.

3. Gurzau D, Caloian B, Frangu F et al. The role of cardiovascular rehabilitation in women with ischemic heart disease and rhythm disorders. Balneo Research Journal. 2018;9:50-53.

4. Pina IL, Balady GJ, Hanson P, et al. Guidelines for clinical exercise testing laboratories: a statement for healthcare professionals from the Committee on Exercise and Cardiac Rehabilitation, American Heart Association. Circulation. 1995;91:912-921.

5. Pop D, Caloian B, Cismaru G. et al. Secondary prevention-an essential component of the comprehensive rehabilitation of patients with heart failure. Balneo Research Journal. 8. 217- 219 .
6. Stein R, Chaitman B, Balady GJ, et al. Safety and utility of exercise testing in emergency room chest pain centers: an advisory from the Committee on Exercise, Rehabilitation, and Prevention, Council on Clinical Cardiology, American Heart Association. Circulation. 2000; 102:1463-1467.

7. Ogawa T, Spina RJ, Martin WH 3rd, et al. Effects of aging, sex, and physical training on cardiovascular responses to exercise. Circulation. 1992;86:494-503.

8. Reddy RK, Pooni R, Zaharieva DP et al. Accuracy of Wrist-Worn Activity Monitors During Common Daily Physical Activities and Types of Structured Exercise: Evaluation Study. JMIR Mhealth Uhealth. 2018 Dec 10;6(12):e10338.

9. Snyder NC, Willoughby CA, Smith BK. Comparison of the Polar V800 and the Garmin Forerunner 230 to Predict VO2max. Above]O2max. J Strength Cond Res. 2019 Jan 10. doi: 10.1519/JSC.0000000000002931.

10. West LR.Strava: challenge yourself to greater heights in physical activity/cycling and running. $\mathrm{Br} \mathrm{J}$ Sports Med. 2015 Aug;49(15):1024.

11. Stragier J, Mechant P, De Marez L et al.ComputerMediated Social Support for Physical Activity: A Content Analysis. Health Educ Behav. 2018 Feb;45(1):124-131. 